\title{
Optical properties and plasmon resonances of titanium nitride nanostructures
}

\author{
M B Cortie, J Giddings and A Dowd
}

University of Technology Sydney

PO Box 123, Broadway NSW 2007 (Australia)

E-mail: michael.cortie@uts.edu.au

PACS : 73.20.Mf, 78.40.-q, 78.67.-n

Short title : Plasmons in titanium nitride nanostructures

\begin{abstract}
We examine the optical properties of nanostructures comprised of titanium nitride, TiN, an electrically conducting intermetallic-like compound. This material can be deposited in the form of durable films by physical vapor deposition. Use of nanosphere templating techniques extend the range of nanostructures that can be produced to include the versatile semi-shell motif. The dielectric properties of $\mathrm{TiN}_{1-x}$ depend upon stoichiometry and are favorable for plasmon resonance phenomena in the mid-visible to near-infrared range of the spectrum and for $x \approx 0$. We analyze the optical phenomena operating in such structures using a combination of experiment and simulation and show that semi-shells of TiN exhibit a tunable localized plasmon resonance with light. The material is however unsuitable for applications in which a long-distance surface plasmon polariton is desired.
\end{abstract}




\section{Introduction}

The field of 'plasmonics' is currently attracting considerable attention, due in part to the many actual or potentially useful applications of plasmon resonances. These extend from spectrally-selective coatings on windows, through various kinds of chemical or biological analytical sensor, to diagnostic and therapeutic medical applications and on to hybrid optoelectronic devices [1-3]. Most of this research is directed at plasmonic nanostructures made of gold. This is primarily because this element has a strong plasmon resonance in the middle of the visible range, and is resistant to oxidation, non-toxic, and readily fabricated into nanostructures $[4,5]$. Actually, a few other elements are also possible candidates for such devices; silver nanospheres, for example, have a very strong plasmon resonance at the blue end of the visible but unfortunately are susceptible to oxidation over a timescale of several days. Nanospheres of lithium, potassium and sodium would also have strong plasmon resonances in the visible $[6,7]$ but of course would be exceedingly rapidly oxidised unless well protected. On the other hand, nanoparticles of copper $[8,9]$, the intermetallic compound $\mathrm{AuAl}_{2}[10]$ and the compounds $\mathrm{VO}_{2}[11,12]$ and $\mathrm{TiN}_{1-x}[13,14]$ exhibit a plasmon resonance too, although it is not necessarily as strong as that of Au or Ag.

Here we consider the merits of $\mathrm{TiN}_{1-x}$ (titanium nitride) as a possible material for plasmonic devices. Unlike gold, titanium nitride is hard and abrasion resistant and has a considerably lower raw material cost. Furthermore, the dielectric properties of $\mathrm{TiN}_{1-\mathrm{x}}$ are very sensitive to its stoichiometry, allowing for a wide tunability in response, if desired. $\mathrm{TiN}_{1-\mathrm{x}}$ has the facecentred cubic crystal structure, and is stable over the range $0<x<0.43$, with $x>0$ corresponding to the situation in which an increasing number of vacancies are substituted in the place of nitrogen atoms in the lattice [15]. It is susceptible to some initial oxidation [13] but this evidently passivates the surface so that further attack does not take place. We will use the term TiN to refer to this compound, irrespective of its stoichiometry, and will describe how 
we prepared both thin films of it and arrays of nanoscale semi-shells. The optical properties and plasmon resonances of these structures were characterized and analyzed, and we conclude with the surprising result that TiN could be a viable selection for devices requiring a localized plasmon resonance.

\section{Experimental}

\subsection{Deposition of TiN coatings and semi-shells}

Monodisperse spherical polystyrene particles of $200 \mathrm{~nm}$ diameter were obtained from Spherotech Inc. The nanospheres were diluted (1:2 v/v) in a solution $(1: 400 \mathrm{v} / \mathrm{v})$ of Triton X100 in methanol. $10 \mu \mathrm{L}$ of the diluted suspension was spun-cast at $2000 \mathrm{rpm}$ onto glass microscope slides to form monolayers of spheres in a hexagonal closest-packed configuration. TiN was deposited by magnetron sputtering of a titanium metal target in a chamber filled with a reduced pressure of $\mathrm{Ar}$ and $\mathrm{N}_{2}$, typically $0.13 \mathrm{~Pa}$ ( $1 \mathrm{mT}$ Torr) of $\mathrm{Ar}$ and $0.03 \mathrm{~Pa}(0.23 \mathrm{mT}$ Torr) of $\mathrm{N}_{2}$. A typical deposition time was 100 seconds, while the applied voltages were in the range 360 to $400 \mathrm{~V}$. Thicknesses of films were monitored using a quartz crystal monitor. For the simple films of TiN on glass we investigated the effect of heating the substrate to $300^{\circ} \mathrm{C}$. However, this was found to be unnecessary as coatings deposited at room temperature also had the very characteristic color and optical properties of TiN. This was fortunate, as deposition onto the polystyrene spheres could obviously only be done at room temperature due to the need to avoid pyrolysis or melting of the polystyrene template. It has also been reported by others that deposition at room temperature is sufficient to produce TiN [16].

\subsection{Characterization}

A sample of the TiN semi-shells formed on the polystyrene spheres was cross-sectioned using focused ion beam (FIB) milling in a FEI Quanta 200 3D workstation using a $20 \mathrm{kV}$ gallium 
ion beam with a current of $0.21 \mathrm{nA}$ impinging on the specimen at 6 degrees from horizontal. The samples were imaged with a Zeiss Supra 55VP scanning electron microscope (SEM). The crystal structure of the film was investigated with X-ray diffraction (XRD) on the powder diffraction beamline at the Australian Synchrotron. A wavelength of $0.1378 \mathrm{~nm}$ was used in the thin film (grazing incidence) geometry where the angle of incidence was fixed at 0.3 degrees so the information depth is expected to be $<80 \mathrm{~nm}$. The diffracted photons were collected with a microstrip detector. Broadening of peaks is dominated by the detector so no attempt was made to analyse microstrain and crystallite size from peak shift and broadening. The dielectric permittivity of TiN coatings was determined by processing transmission and reflection data using the WVASE32 software from J. A. Woollam Co., Inc.

\subsection{Numerical simulations of the optical properties of TiN nanostructures}

Optical properties were simulated using the DDSCAT program of Draine and Flatau [17, 18], with some added coding by one of the present authors (M.C.) to permit the input of periodic structures of arbitrary geometry. The DDSCAT program invokes the discrete dipole approximation (DDA) in which the target nanostructure is discretized into an array of point dipoles. The interaction of the array of point dipoles with a monochromatic light source is then solved numerically. The DDA has been widely tested and found to be accurate [19-22], provided a sufficiently fine mesh is used to describe the target. Of course, practical limitations on computing resources do limit the dipole spacing that can be used. In particular, calculations involving large values of $m$, the complex refractive index, require very small dipole spacings and long computation times to converge. Here a dipole spacing of $10 \mathrm{~nm}$ was found to be a reasonable compromise, equivalent to representing the semi-shell with about 250,000 dipoles. This is a bigger dipole than used for gold or silver but is satisfactory here because the complex refractive index of $\mathrm{TiN}$ is smaller than that of the precious metals. 
The simulated semi-shell has a core diameter of $200 \mathrm{~nm}$, and a coating thickness of $30 \mathrm{~nm}$ at its apex, tapering down to zero at the edges. Surface roughness was simulated using a Monte Carlo-style technique in which up to 4000 small hemispheres of TiN were randomly thrown down onto the core and allowed to fuse together. These geometric parameters were selected by examination of the experimentally produced semi-shells. However, the actual semi-shells did not taper to quite as sharp a rim as the simulated ones. The simulated targets were rendered to a dipole array for the actual calculations.

\section{Results}

We will first describe the properties of the smooth deposits of TiN directly onto glass substrates before moving on to the more complex behaviour of the arrays of TiN semi-shells.

\subsection{Properties of smooth deposits of TiN on glass}

The deposits of TiN on glass were golden-colored, which is the characteristic color of this compound. X-ray diffraction using synchrotron radiation at a grazing angle of incidence indicated the lattice parameter to be about $0.42 \mathrm{~nm}$, which is comparable to the $0.424 \mathrm{~nm}$ reported in the literature [13]. The diffraction pattern from a TiN thin film on a glass substrate is shown in figure 1 . The broad peak at $2 \theta=25^{\circ}$ is due to the silica of the underlying substrate, while all other peaks correspond to the cubic phase of TiN. The peaks have been broadened by the grazing angle incidence geometry used and, possibly, by an ultra-fine crystallite size. The assignment of reflections to TiN index planes is indicated. No additional peaks are observed, indicating the phase purity of these films. The $\{220\}$ peak is somewhat stronger than it would have been in a random sample, suggesting that there is a degree of crystallographic texture in the films. Imaging using both an atomic force and a scanning electron microscope indicated that the upper surface of the deposit was characterized by flat 
mounds of about 1 or $2 \mathrm{~nm}$ height, and several nanometers diameter, figure 2 . This morphology may explain the presence of the $\mathrm{SiO}_{2}$ background in the XRD pattern since an estimation of the depth sampled in the XRD pattern in figure 1 would yield only $20 \mathrm{~nm}$ if based on perfectly smooth and dense TiN. However, the particular film was estimated to be of the order of $30 \mathrm{~nm}$ thick on the basis of its optical properties, so there should have been almost no $\mathrm{SiO}_{2}$ signal. The discrepancy is explained if a lower density and some surface roughness of the film are taken into account. In this case the penetration depth may be estimated to be about $85 \mathrm{~nm}$ from the expression

$$
z=\sin (\phi) / \mu
$$

where $z$ is the penetration depth, $\phi$ the angle of the X-ray beam from the plane of the sample, and $\mu$ the linear attenuation coefficient.

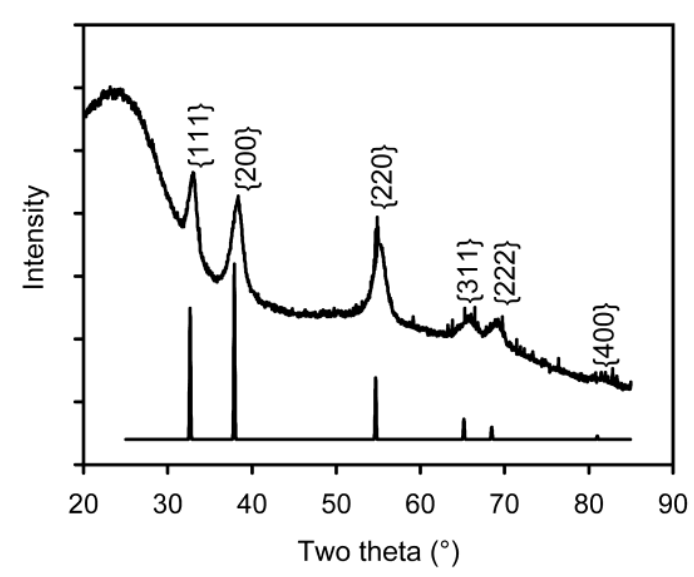

Figure 1. X ray diffraction pattern of thin film of TiN on glass (Sample 1), obtained using synchrotron radiation of $0.1378 \mathrm{~nm}$. A calculated pattern for $a=0.4244 \mathrm{~nm}$ is shown below the measured pattern. No attempt was made to subtract the background from the measured data. 


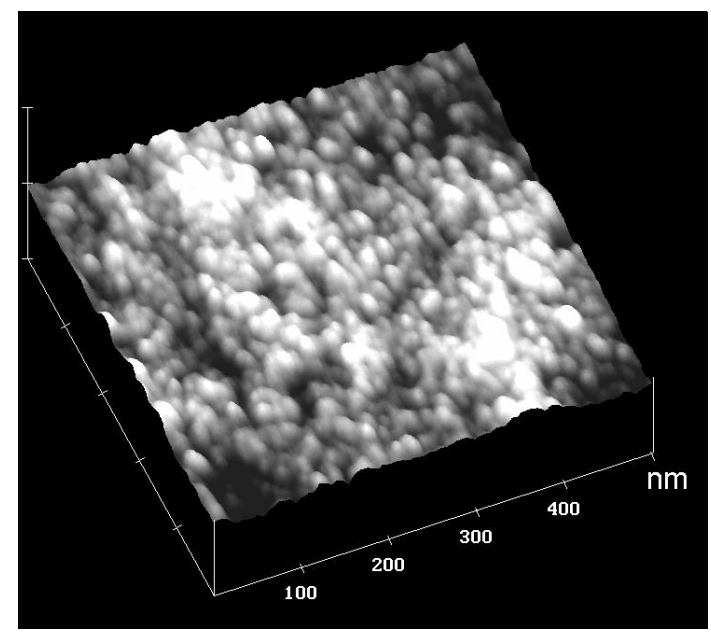

(a)

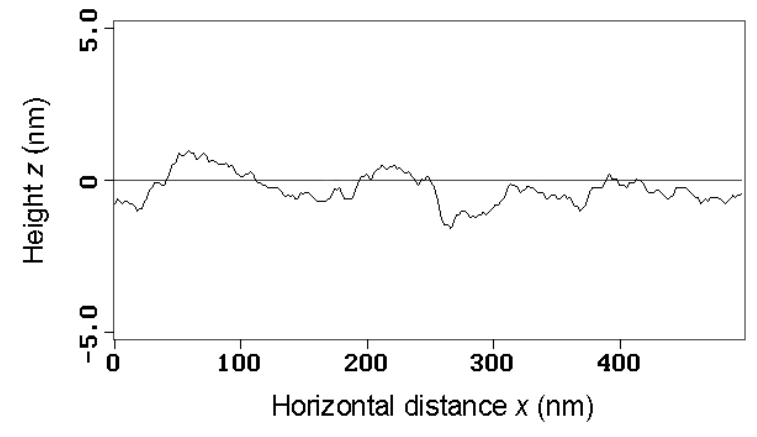

(b)

Figure 2. Morphology of TiN thin films on glass. (a) Surface topography of Sample 1, taken with atomic force microscope. (b) Profile across a portion of the surface shown in (a).

The optical properties of the thin films are compared in figure 3. The pronounced transmission maximum occurs at $\sim 480 \mathrm{~nm}$ in the thinnest films, decreasing to $\sim 430 \mathrm{~nm}$ in the thickest films. It is clear that the dominant effect overall is the thickness of the coating. The transmission maximum can be matched to the extinction minimum reported by Reinholdt et al. for aggregates of TiN nanoparticles at 400 to $420 \mathrm{~nm}(3.0$ to $3.1 \mathrm{eV})$ [13], and to the minimum in reflectance shown in figure $3(\mathrm{~b})$. TiN is comparatively absorptive at wavelengths $<400 \mathrm{~nm}$ due, apparently, to interband transitions [13]. In contrast, the spectra in figure 3(b) indicate that the film reflects infra-red wavelengths, as expected from a metallic conductor. For films of $70 \mathrm{~nm}$ or thicker the reflectance in the infrared is essentially independent of thickness. However, Films A and B are so thin that a large proportion of the light is actually transmitted, with a corresponding decrease in reflectance ( $c f$. figure 3(a)). 


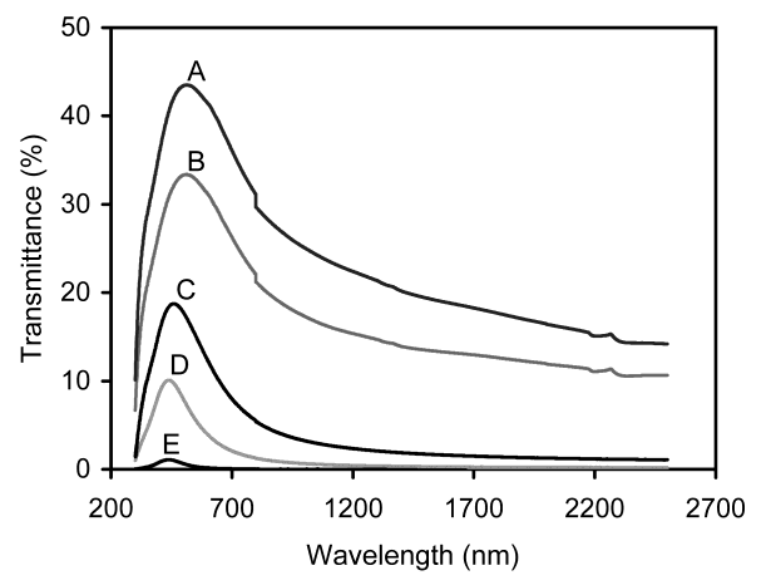

(a)

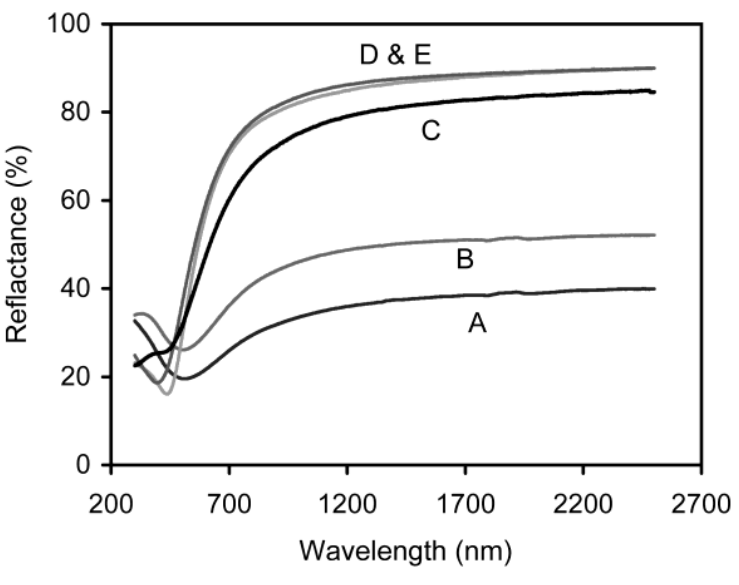

(b)

Figure 3. Optical properties of thin films of TiN on glass. Films $A$ to $E$ are of increasing thicknesses deposited on a substrate heated to $300^{\circ} \mathrm{C}$. Thickness of TiN layer estimated from analysis of optical transmission, $A=22 \mathrm{~nm}, B=30 \mathrm{~nm}, C=50 \mathrm{~nm}, D=70 \mathrm{~nm}$ and $E=139 \mathrm{~nm}$. (a) Transmittance spectra. The small steps in the data at $\sim 800$ and $\sim 2300 \mathrm{~nm}$ are measurement artifacts. (b) Reflectance spectra.

The dielectric permittivities obtained for the present samples are plotted in figure 4 and compared with previous studies in the literature. It can be seen that coatings deposited at room temperature had similar optical properties to those produced at $300^{\circ} \mathrm{C}$ and the data lie within the range of values published by Fuentes et al. [23] and Jeyachandran et al. [16] for sputtered films. Of particular importance is that $\varepsilon_{1}$ becomes negative for $\lambda>476 \mathrm{~nm}$, and equals -2 at about $550 \mathrm{~nm}$. In general, a plasmon resonance will occur in a nanosphere when $\varepsilon_{1} \approx-2$, provided that $\varepsilon_{2}$ is not too large. This may be seen in simulations of the optical properties of discrete nanoparticles made using the present dielectric property data, figure 5 . In the case we have simulated a nano-cube of $\mathrm{TiN}$, rather than a nanosphere, because this is characteristic morphology of its nano-crystal. The resonance criterion of isolated cubes of TiN of 10 to 50 $\mathrm{nm}$ size is met at about $560 \mathrm{~nm}$. The resulting extinction peak increases in intensity, and redshifts slightly, as the size of the cube is increased. These predictions can be contrasted to the measured data of Reinholdt et al., who found a plasmon absorption in the range 700 to 750 $\mathrm{nm}$ for aggregates of $8 \mathrm{~nm}$ TiN cubes immersed in a matrix of dielectric oxides. In this case 
the red-shifted absorption of Reinholdt et al.'s material is probably due to the combined effects of the dielectric matrix and agglomeration of the individual nanoparticles.

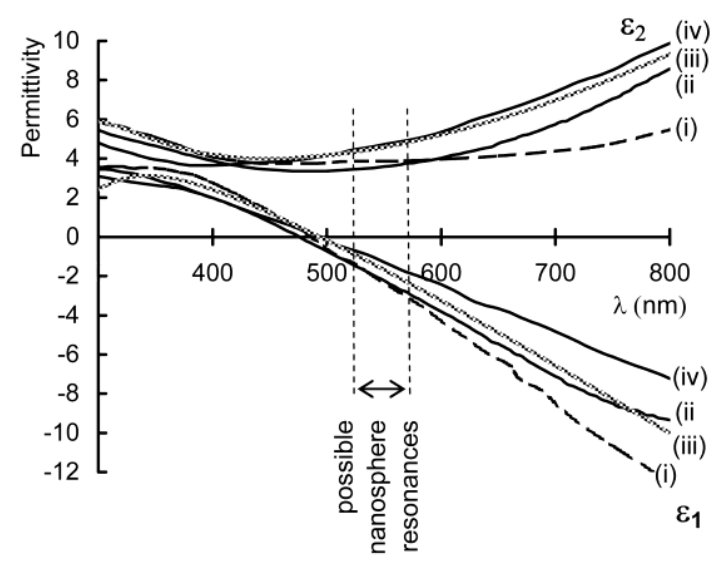

Figure 4. Dielectric permittivity of the TiN coatings produced in the present work, compared to data from Fuentes et al.[23] and Jeyachandran et al.[16]. Key: (i) Fuentes et al, (ii) present work, deposited at $300^{\circ} \mathrm{C}$, (iii) present work, deposited at room temperature, (iv)

Jeyachandran et al.

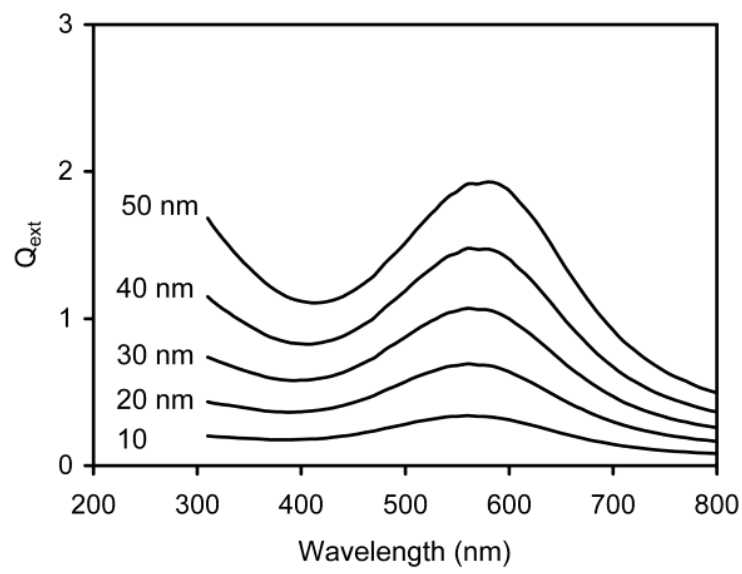

Figure 5. Predicted optical extinction spectra of isolated TiN cubes of between 10 and $50 \mathrm{~nm}$ in size. The peak between 500 and $600 \mathrm{~nm}$ is due to a plasmon resonance.

\subsection{Properties of arrays of TiN semi-shells on polystyrene cores}

3.2.1 Measured data. Figure 6 shows a typical structure obtained by TiN deposition on selfassembled colloidal crystals of polystyrene spheres. The TiN semi-shells have a rough upper surface, figure 6(b). The cross-sectional profile of the semi-shells was investigated by doublebeam focused ion beam milling. It is evident that these semi-shells would be thickest on their 
uppermost surface, and thin out towards their equators, figure 6(c). It is also worth noting that the polystyrene immediately under the shells has apparently disappeared, to leave a cavity. This may be an artifact caused by the milling process, but the possibility also arises that sufficient energy was released during the deposition of TiN that the polystyrene immediately below the coating was destroyed. The transmittance of the reference thin film made at the same time as the semi-shells shown in figure 6(c) indicated a film thickness of 40 to $41 \mathrm{~nm}$. In contrast, the sections shown in figure 6(c) are about 30 to $35 \mathrm{~nm}$ in thickness, but these are already some way down towards the equatorial girth of the semi-shells and would therefore be less than the maximum coating thickness. The tapered profile was incorporated into the geometric model of the targets used in the numerical simulations of optical properties.

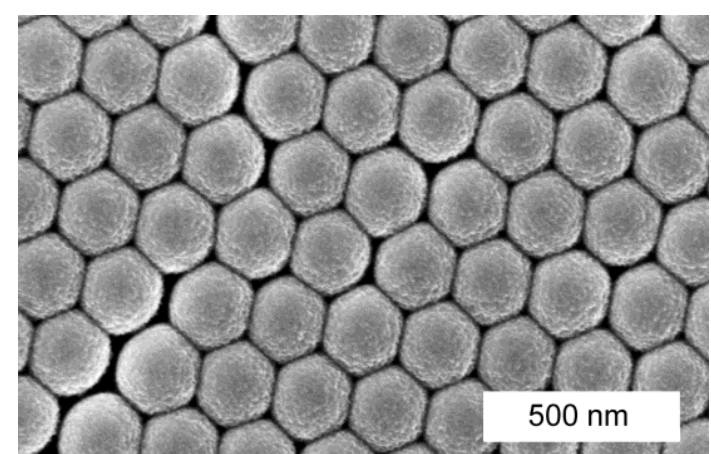

(a)

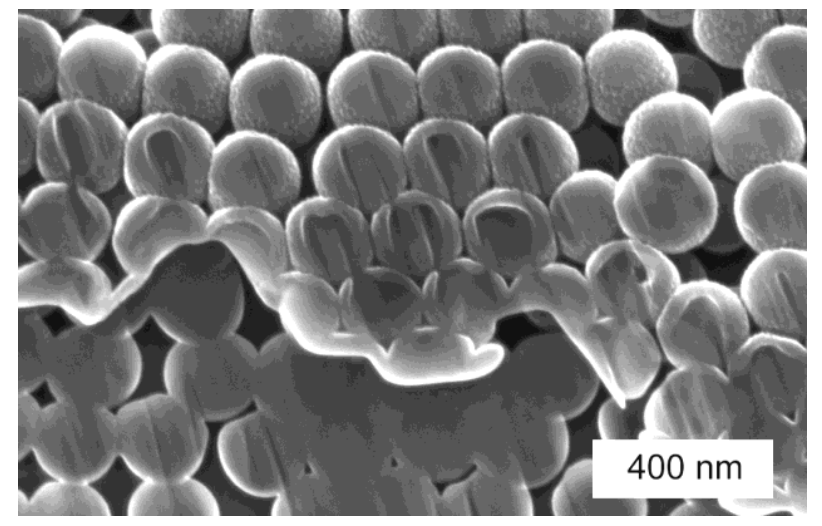

(c)

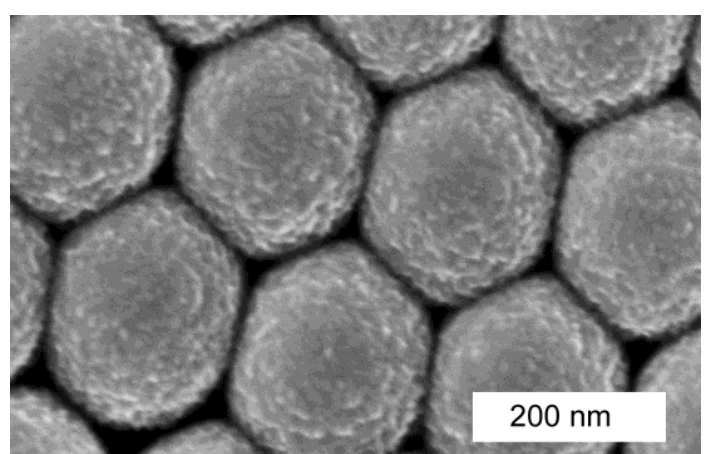

(b)

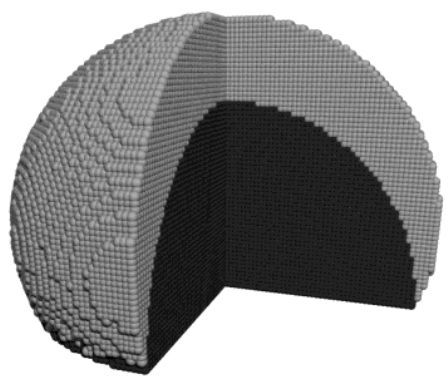

(d)

Figure 6. Titanium nitride semi-shells. (a) Scanning electron microscope image of array of TiN semishells formed on a colloidal crystal monolayer of polystyrene nanospheres. (b) Highmagnification view of TiN deposit, showing surface roughness. (c) Cross-sections taken through TiN semi-shells using focused ion beam milling. (d) Example of dipole model of TiN semi-shell with $200 \mathrm{~nm}$ internal diameter and a maximum coating thickness of $40 \mathrm{~nm}$, with associated polystyrene core. Here, a section has been cut out of the model to show the tapered structure. 
The reflectance and transmittance of an array of TiN semi-shells are compared to that of a plain TiN film in figure 7 . Both the array and the plain samples had the same nominal coverage of TiN, produced by identical chamber deposition conditions. It can be seen that the big difference in optical properties between the two sample geometries is in reflectivity, with that of the plain TiN film being significantly higher than that of the semi-shell array, particularly at higher wavelengths.

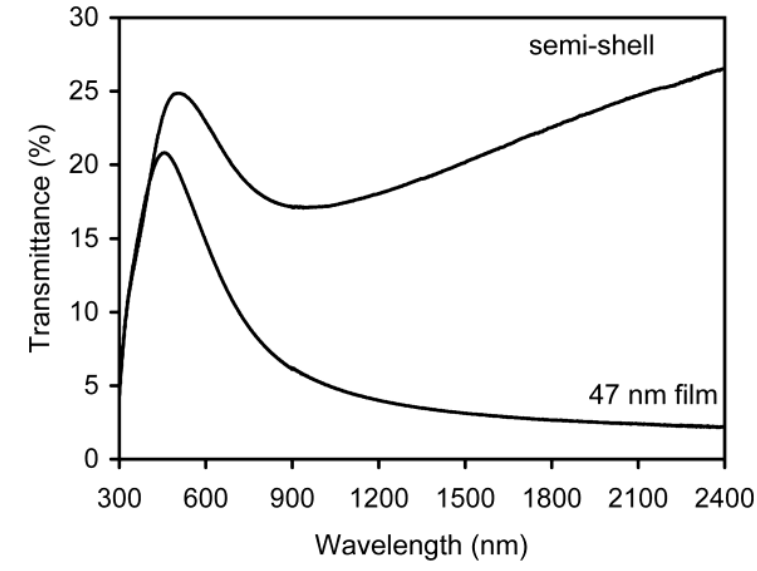

(a)

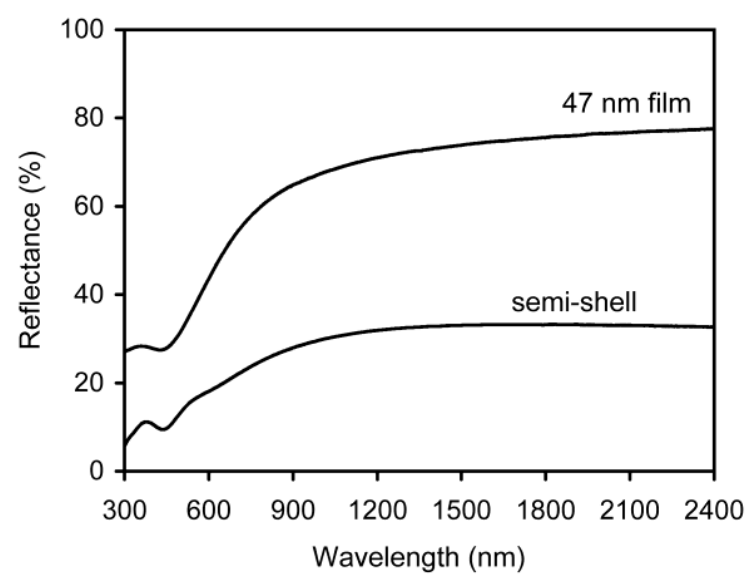

(b)

Figure 7. Optical properties of arrays of TiN semi-shells compared to those of a thin film of TiN deposited under the same conditions.( a) Transmittance. (b) Reflectance.

\subsubsection{Numerical simulations of optical properties of semi-shell. The TiN of the real semi-}

shells partially encloses a core of polystyrene, with a refractive index of about 1.58 . In principle, the effect of this should be to red-shift any localized surface plasmon resonance on the TiN semi-shell. Therefore, it was considered desirable to include the effect of the core in the numerical simulations, however the degree of physical contact between the shell and the original polymer core was uncertain. Figure 8 shows the extinction efficiency of a single semi-shell (with core) for light propagating parallel to the axis of rotational symmetry of the semi-shell, and for varying degrees of contact between core and TiN coating ( $R$ full contact, $P$ and $Q$ with an air gap). Also shown is a semi-shell $\left(P^{\prime}\right)$ with a roughened surface texture. The maximum thickness of coating in this simulation was $40 \mathrm{~nm}$, which matches the thickness of 
the coatings of the planar thin films deposited under identical conditions. Also shown is the measured extinction of two semi-shell arrays of similar thickness, where extinction is the sum of reflection and absorption. There is a strong extinction minimum between 470 and $485 \mathrm{~nm}$, and a very broad extinction peak at about $920 \mathrm{~nm}$. These can be correlated with the minimum and maximum in the calculated data, provided that it is accepted that some shrinkage of the polystyrene core away from the TiN shell has occurred, as discussed previously. The electric field associated with the resonance at $950 \mathrm{~nm}$ is shown in figure 8(c) (an animation is also provided in the Supporting Information). The electric field of the light is perpendicular to the axis of rotational symmetry of the semi-shell in these simulations. The resonance associated with this orientation has been previously designated the $\beta$ resonance to differentiate it from the other possibility, the $\alpha$ resonance, in which the electric field is parallel to the axis of rotational symmetry [24], figure $8(d)$. 


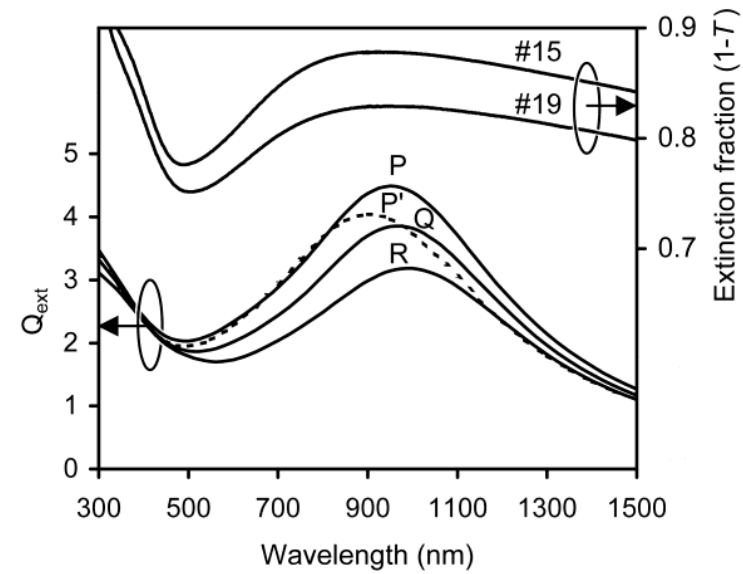

(a)

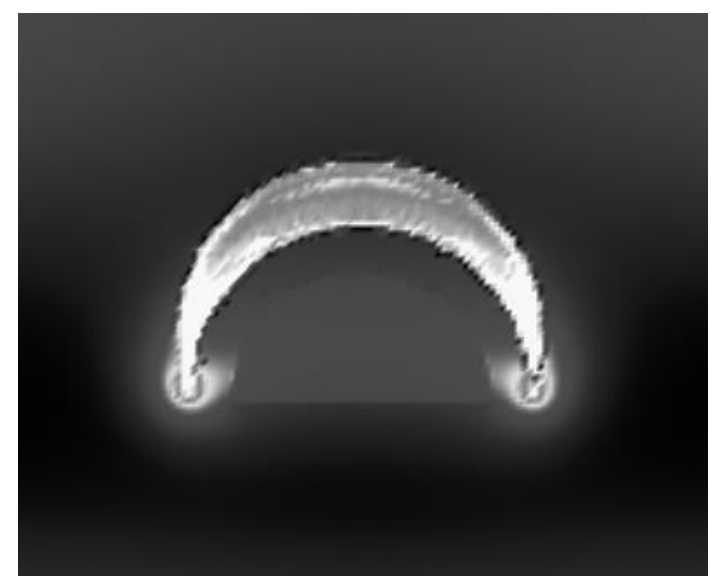

(c)
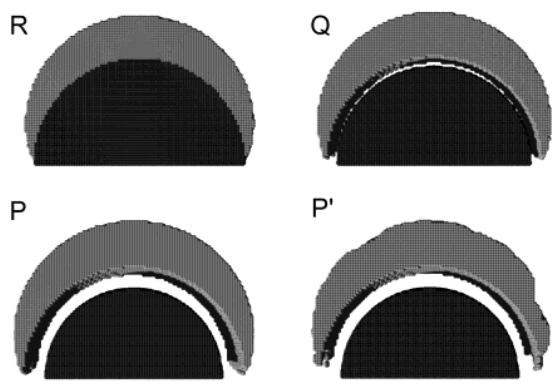

(b)
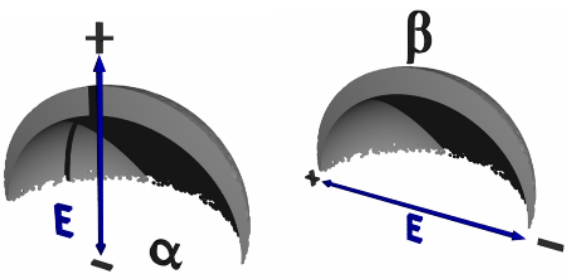

(d)

Figure 8. Optical properties of single semi-shells. (a) Comparison of measured (\#15 and \#19) and simulated $\left(P, P^{\prime}, Q\right.$ and $\left.R\right)$ extinction efficiencies. The dashed line $\left(P^{\prime}\right)$ shows a rough semi-shell with the same degree of contact as $P$. (b) Cross-sections through simulation targets depicted in (a). (c) Electric field calculated for excitation of target $P$ at $950 \mathrm{~nm}$. (d) Nature of $\alpha$ and $\beta$ plasmon resonances in semi-shells.

Another factor to consider is that the semi-shells in these samples are not isolated from one another and instead form closely-packed arrays. While simulation of an infinitely periodic hexagonal array would have exceeded the capability of the available software (due to the very large number of dipoles required) further insight could be gained here by calculation of the optical properties of infinitely periodic chains of semi-shells. Two further types of $\beta$ resonance are possible, figure 9 . Here we denote the three kinds of $\beta$ resonance as $\beta_{1}$ (for isolated nanoparticles), $\beta_{2}$ (electric field across the chain) and $\beta_{3}$ (electric field along the chain). Obviously, if the polarization of light is at angle between $\beta_{2}$ and $\beta_{3}$ with the chain then 
both resonances can be excited, in proportion to the resolved components of $E$ in each direction. The effect of including the resonances in infinite chains of these semi-shells is examined in figure 10 , in which the $\beta_{2}$ and $\beta_{3}$ resonances are shown as dashed lines. Clearly, the $\beta_{2}$ resonance is not very different from the $\beta_{1}$ resonance of a single nanoparticle, whereas the extinction peak for the $\beta_{3}$ resonance lies in the infra-red, and beyond the reach of the present simulations. We submit that the situation in the actual semi-shell arrays is likely to contain aspects of both the $\beta_{2}$ and $\beta_{3}$ resonances shown here, albeit somewhat modified by the hexagonal symmetry of the array. A qualitative depiction of the possible resulting resonance was obtained by performing a weighted average on the two resonances (solid line). This is a purely illustrative average provided to suggest how the measured trends might be explained by a mixture of $\beta$ resonances; a more rigorous depiction would require a more detailed geometric model which is beyond the scope of the present work. 

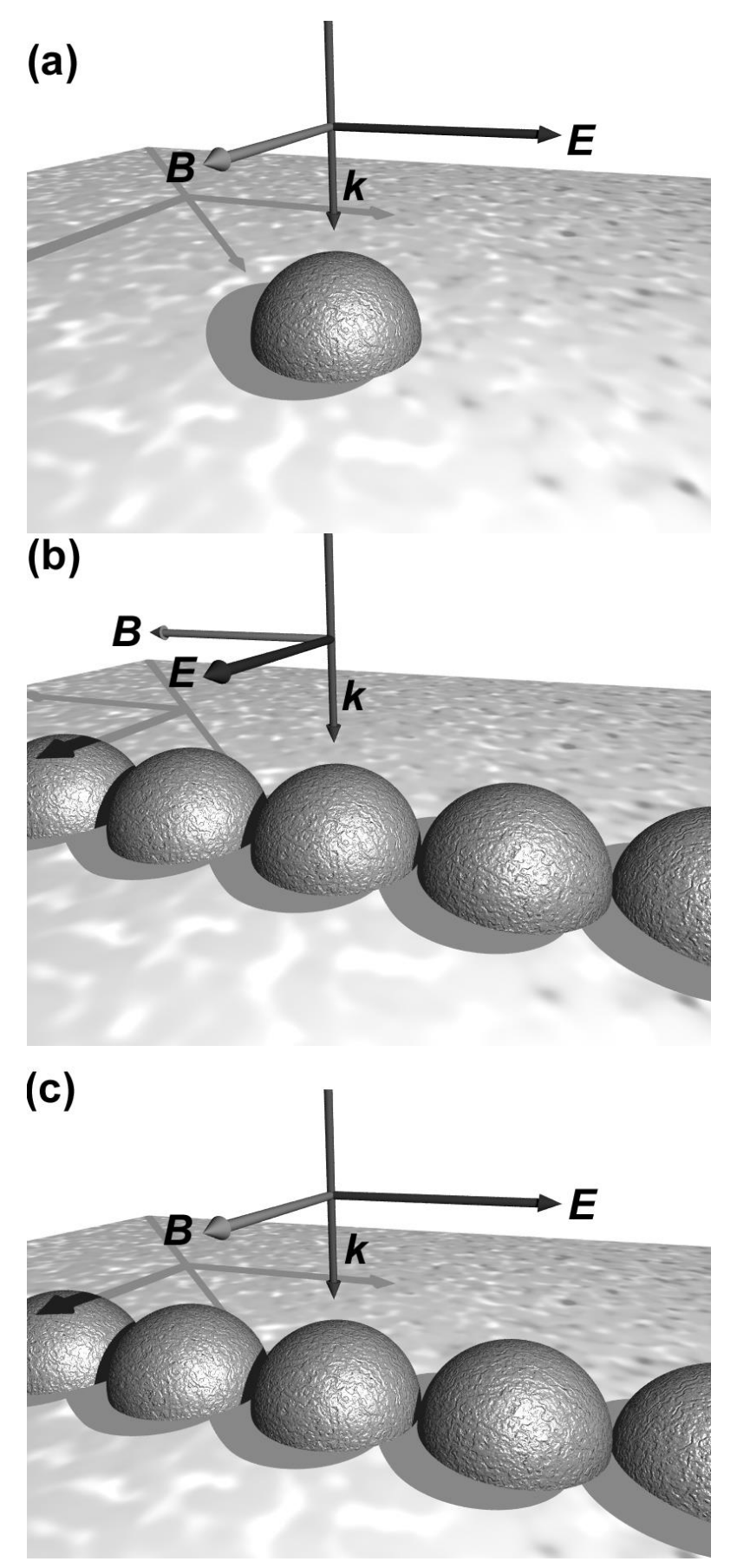

Figure 9. Three scenarios for the $\beta$ (longitudinal) resonance in semi-shells, $\boldsymbol{k}$ is the direction of propagation of the light, $\boldsymbol{E}$ is the direction of the electric field, and $\boldsymbol{B}$ is the direction of the magnetic field. (a) $\beta_{1}$ resonance of an isolated semi-shell. (b) $\beta_{2}$ resonance of infinite chain of semi-shells. (c) $\beta_{3}$ resonance of infinite chain of semi-shells. 


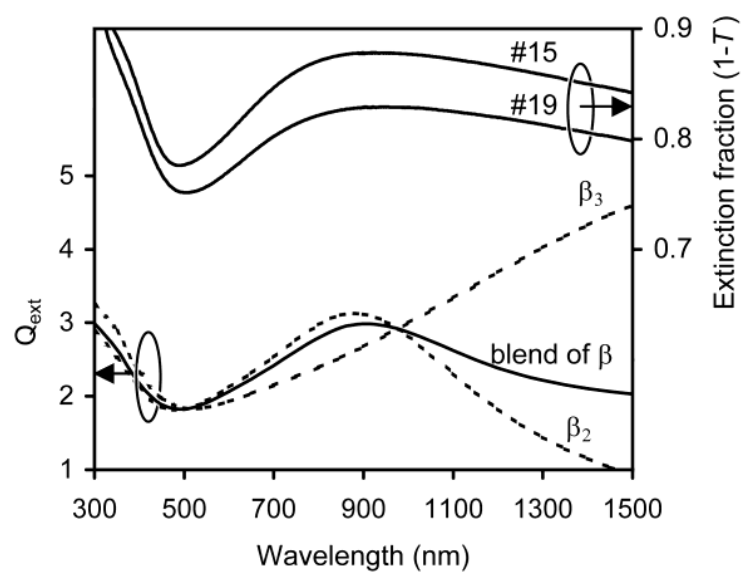

Figure 10. Simulations of the optical extinction properties of infinite 1-D arrays of $200 \mathrm{~nm}$ inside diameter, $280 \mathrm{~nm}$ outside diameter TiN semi-shells, compared to the measured extinction fraction. Also shown is a merely illustrative composite curve obtained by averaging the effects of the $\beta_{2}$ and $\beta_{3}$ resonances.

In previous work on Ag semi-shells, it was found that the optical properties could not be explained in terms of $\beta$ resonances, and evidence was presented for a coupling of light into the plane of the semi-shell array and excitation of the $\alpha$ resonance [25]. This phenomenon does not appear to occur in the arrays of TiN semi-shells, due probably to the relatively high damping in TiN relative to that in Ag. Therefore, any surface plasmon polaritons that couple into the plane of the array of semi-shells would be rapidly attenuated. We will return to this point in the next section.

\section{Discussion of the suitability of TiN for plasmonic devices}

There are two somewhat disparate branches of the field of 'plasmonics': localised surface plasmon resonances in discrete, nanoscale, particles, and propagating surface plasmon polaritons (SPPs) in microscopic structures [26]. In either case, the intensity of the plasmon is determined by a range of factors including dielectric permittivity, geometry, surface roughness, and surrounding medium (if any). However, the complex dielectric permittivity, $\varepsilon$, given by 


$$
\varepsilon=\varepsilon_{1}+i \varepsilon_{2}
$$

is the starting consideration. For resonance to occur the material must be metallic, so that $\varepsilon_{1}<0$. Furthermore, $\varepsilon_{2}$ should be as small as possible because damping of the resonance is approximately in proportion to it. In figure 11 we plot the measured values of $\varepsilon_{1}$ and $\varepsilon_{2}$ of the present TiN and compare them to published values for a range of other materials. For localised plasmon resonances the important region is $-4<\varepsilon_{1}<-1$. Arnold and Blaber have shown that it is useful to consider $\varepsilon_{1}$ as an independent variable which determines the type of application possible [27]. For example, flat plasmonic lenses require $\varepsilon_{1}=-1$, nanosphere resonances in vacuum require $\varepsilon_{1}=-2$, while rod or shell resonances are possible out to $\varepsilon_{1}$ values of as negative as -30 . The value of $\varepsilon_{1}$ necessary for a particular resonance does also depend on the permittivity of the surrounding medium, $\varepsilon_{\mathrm{m}}$, as shown by the dashed lines on the figure, drawn for a nanosphere resonance. The damping at each of these values of $\varepsilon_{1}$ is substantially determined by the corresponding value of $\varepsilon_{2}$. It can be seen that $\mathrm{TiN}$ is comparable to $\mathrm{Cu}$ over the range of $\varepsilon$ from -5 to -1 , which corresponds to wavelengths of light between 653 and $528 \mathrm{~nm}$ respectively for the TiN, but 481 and $305 \mathrm{~nm}$ respectively for $\mathrm{Cu}$. In general, it would be better to use $\mathrm{TiN}$ at $\varepsilon_{1} \approx-1$, ie. corresponding to the shorter wavelengths in the range given because this value is correlated with the lowest possible value of $\varepsilon_{2}$. The opposite situation applies in the case of $\mathrm{Au}$ and $\mathrm{Cu}$, where more negative values of $\varepsilon_{1}$ (corresponding to longer wavelengths of light) are advantageously correlated with smaller magnitudes of $\varepsilon_{2}$. Put another way, unlike TiN, $\mathrm{Au}$ and $\mathrm{Cu}$ become increasingly better plasmonic resonators as the plasmon resonance is red-shifted into the upper visible or nearinfrared. 


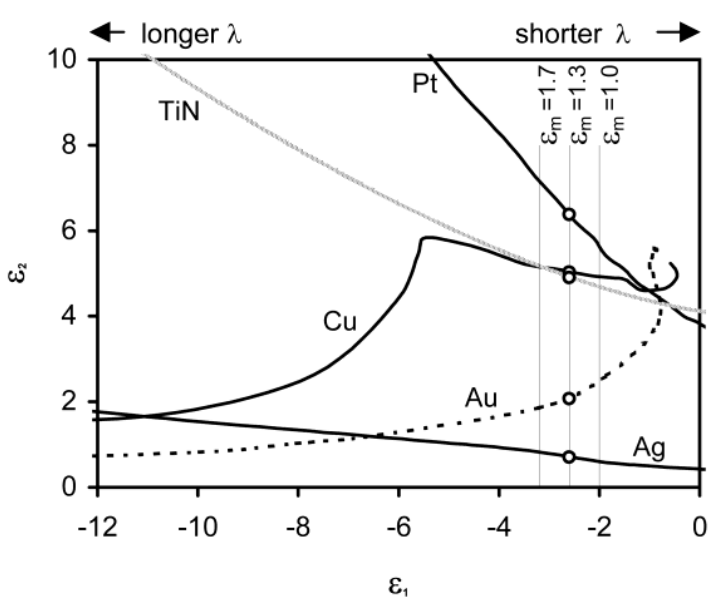

Figure 11. The complex dielectric permittivity of selected materials compared in the region of the spectrum in which their $\varepsilon_{1}$ passes through -2 . The plasmon resonance of a nanosphere is excited at increasingly more negative values of $\varepsilon_{1}$ as the permittivity of the surrounding medium is increased from 1.0 (vacuum) to 1.7 (thin lines). This corresponds to longer wavelengths of light, ie. a red-shift in the resonance.

The merits of using TiN in the applications involving SPPs can be partially determined by simply calculating the distance over which the SPP would propagate along a TiN/vacuum interface, and comparing it to other materials. Here we have used the expression [28]

$$
L_{S P P}=\lambda_{0} \frac{\varepsilon_{1}^{2}}{2 \pi \varepsilon_{2}}
$$

where $L_{\mathrm{SPP}}$ is the distance over which the power of the SPP falls to 1/e of its starting value and $\lambda_{0}$ is the wavelength of the light that excited the SPP. Clearly SPPs will propagate further for more negative values of $\varepsilon_{1}$, and smaller values of $\varepsilon_{2}$. The results are shown in figure 12 . It is obvious that SPPs on a TiN surface would be very rapidly damped compared to those on a surface of $\mathrm{Au}, \mathrm{Ag}$, or even $\mathrm{Cu}$. TiN is apparently not a good choice of material for an application requiring a strong SPP. 


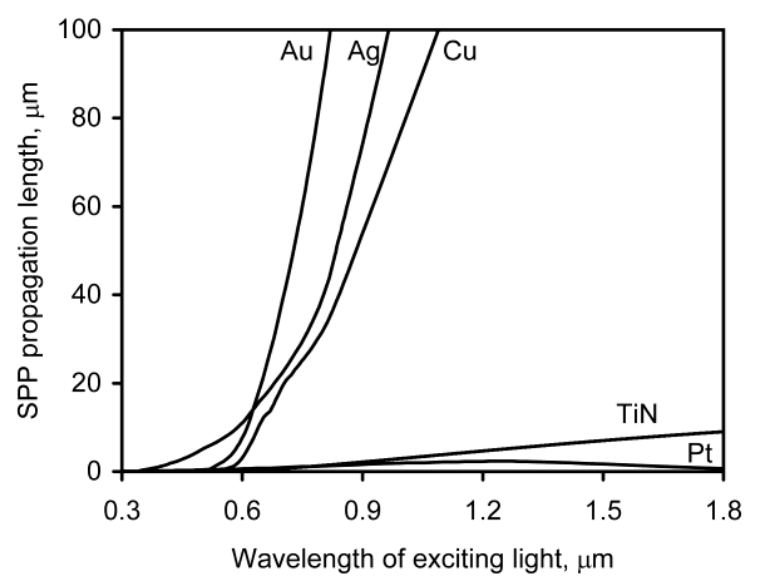

Figure 12. Approximate distance of propagation of surface plasmon polaritons on surfaces of the indicated materials (in vacuum).

\section{Conclusions}

We have shown here that it is feasible to design and fabricate plasmonically-active structures using titanium nitride (TiN). Discrete nanoparticles and periodic arrays of this material will exhibit strong localized surface plasmons, however propagation of surface plasmon polaritons on planar surfaces is expected to be very limited in extent. The semi-shells can sustain localized plasmon resonances, the position of which can furthermore be adjusted by control of geometric parameters. Although the intensity of the resonances in TiN are not as great as those in, for example, gold, the raw material cost of TiN is considerably lower. Furthermore, TiN itself is hard and comparatively durable under atmospheric conditions, unlike some of the other alternative candidates for plasmonic applications such as $\mathrm{Ag}, \mathrm{Al}$ or the alkali metals, although this advantage in respect of mechanical properties may be attenuated if $\mathrm{TiN}$ is used in conjunction with a soft substrate.

\section{Acknowledgments}

We thank K. Van't Schip for assisting with the determination of the dielectric permittivity of our samples, R. Wuhrer for scanning electron microscopy, and the Electron Microscopy Unit at the University of Sydney for focused ion beam milling. Part of this research was 
undertaken on the Powder Diffraction Beamline at the Australian Synchrotron, Victoria, Australia. The views expressed herein are those of the authors and are not necessarily those of the owner or operator of the Australian Synchrotron.

\section{Supporting Information}

AVI movie showing magnitude and location of electric fields excited on a single titanium nitride semi-shell with $950 \mathrm{~nm}$ light.

\section{References}

[1] Ozbay, E. 2006 Science 311(5758) 189.

[2] Pissuwan, D., Valenzuela, S. and Cortie, M. B. 2006 Trends Biotechnol. 24(2) 62.

[3] Schelm, S. and Smith, G. B. 2003 Appl. Phys. Lett. 82(24) 4346.

[4] Stokes, N., McDonagh, A. M. and Cortie, M. B. 2007 Gold Bull. 40(4) 310.

[5] Cortie, M. B. 2004 Gold Bull. 3712.

[6] Blaber, M. G., Arnold, M. D., Harris, N., Ford, M. J. and Cortie, M. B. 2007 Physica B-Condensed Matter 394(2) 184.

[7] Blaber, M. G., Arnold, M. D. and Ford, M. J. 2009 J. Phys. Chem. C 1133041.

[8] Salzemann, C., Lerme, J., Urban, J. and Lisiecki, I. 2005 Chem. Mater. 171279.

[9] Cortie, M. B., Xu, X. and Ford, M. J. 2006 Phys. Chem. Chem. Phys. 83520.

[10] Supansomboon, S., Maaroof, A. and Cortie, M. B. 2008 Gold Bull. 41(4) 296.

[11] Bai, H., Cortie, M. B., Maaroof, A. I., Dowd, A., Kealley, C. and Smith, G. B. 2009 Nanotechnology 20 article 085607.

[12] Lopez, R., Haynes, T. E., Boatner, L. A., Feldman, L. C. and R. F. Haglund, J. 2002 Opt. Lett. 27(15) 1327.

[13] Reinholdt, A., Pecenka, R., Pinchuk, A., Runte, S., Stepanov, A. L., Weirich, T. E. and Kreibig, U. 2004 Eur. Phys. J. D 3169.

[14] Hibbins, A. P., Sambles, J. R. and Lawrence, C. R. 1998 J. Modern Optics 45(10) 2051.

[15] Köhler, K., Geserich, H. P. and Christensen, A. N. 1986 Z. Phys. B. Condensed Matter 62319.

[16] Jeyachandran, Y. L., Narayandass, S. K., Mangalaraj, D., Areva, S. and Mielczarski, J. A. 2007 Mater. Sci. Eng., A 445-446 223.

[17] Draine, B. T. and Flatau, P. J. 1994 J. Opt. Soc. Am. A 11(4) 1491.

[18] Draine, B. T. and Flatau, P. J. 2008, User Guide for the Discrete Dipole Approximation Code DDSCAT 7.0, http://arxiv.org/abs/0809.0337., accessed September 2008.

[19] Brioude, A., Jiang, X. C. and Pileni, M. P. 2005 J. Phys. Chem. B 10913138.

[20] Kelly, K. L., Coronado, E., Zhao, L. L. and Schatz, G. C. 2003 J. Phys. Chem. B 107 668 . 
[21] Myroshnychenko, V., Rodríguez-Fernández, J., Pastoriza-Santos, I., Funston, A. M., Novo, C., Mulvaney, P., Liz-Marzán, L. M. and Abajo, F. J. G. d. 2008 Chem. Soc. Rev. 37 1792

[22] Yurkin, M. A. and Hoekstra, A. G. 2007 J. Quant. Spectrosc. Radiat. Transfer 106 558.

[23] Fuentes, G. G., Elizalde, E. and Sanza, J. M. 2001 J. App. Phys. 90(6) 2737.

[24] Cortie, M. B. and Ford, M. J. 2007 Nanotechnology 18(23).

[25] Maaroof, A. I., Cortie, M. B., Harris, N. and Wieczorek, L. 2008 Small 4(12) 2292.

[26] Maier, S. A. 2007 Plasmonics. Fundamentals and Applications, (New York: Springer).

[27] Arnold, M. D. and Blaber, M. G. 2009 Opt. Exp. 17(5) 3835.

[28] Barnes, W. L. 2006 J. Opt. A: Pure Appl. Opt. 8 S87. 